\title{
Prevalence and molecular characterization of pyrazinamide resistance among multidrug-resistant Mycobacterium tuberculosis isolates from Southern China
}

\author{
Yu Pang ${ }^{1 \dagger}$, Damian Zhu ${ }^{2 \dagger}$, Huiwen Zheng ${ }^{3}$, Jing Shen ${ }^{2}$, Yan Hu${ }^{2}$, Jie Liu ${ }^{2^{*}}$ and Yanlin Zhao ${ }^{3^{*}}$
}

\begin{abstract}
Background: Pyrazinamide (PZA) plays a unique role in the treatment for multidrug-resistant tuberculosis (MDR-TB) in both first- and second-line regimens. The aim of this study was to investigate the prevalence and molecular characterization of PZA resistance among MDR-TB isolates collected in Chongqing municipality.

Methods: A total of 133 MDR-TB isolates were collected from the smear-positive tuberculosis patients who were registered at local TB dispensaries of Chongqing. PZA susceptibility testing was determined with a Bactec MGIT 960 system. In addition, the genes conferring for PZA resistance were screened by DNA sequencing.

Results: Of these 133 MDR-TB isolates, 83 (62.4\%) were determined as PZA-resistant by MGIT 960. In addition, streptomycin- $(83.1 \%$ vs. $56.0 \%, P<0.01)$, ofloxacin- (51.8\% vs. $18.0 \%, P<0.01)$, kanamycin- $(22.9 \%$ vs. $2.0 \%, P<0.01)$, amikacin- $(18.1 \%$ vs. $2.0 \%, P=0.01)$, capromycin-resistance $(12.0 \%$ vs. $2.0 \%, P=0.05)$, were more frequently observed among PZA-resistant isolates compared with PZA-susceptible isolates. Sequence analysis revealed that 73 out of 83 (88. 0\%) MDR strains harbored a mutation located in the pncA gene, including $55(75.3 \%, 55 / 73)$ of single nucleotide substitutions and $18(24.7 \%, 18 / 73)$ of frameshift mutation, while no genetic mutation associated with PZA resistance was found in the rps $A$ gene. The pncA expression of strains harboring substitution from $A$ to $G$ at position -11 in the promoter region of pncA was significantly lower than that of H37Rv $(P<0.01)$.
\end{abstract}

Conclusions: In conclusion, our data have demonstrated that the analysis of the pncA gene rather than rpsA gene provides rapid and accurate information regarding PZA susceptibility for MDR-TB isolates in Chongqing. In addition, loss of pncA expression caused by promoter mutation confers PZA resistance in MDR-TB isolates.

\section{Background}

Multidrug resistant tuberculosis (MDR-TB) is a major concern hampering global tuberculosis control efforts $[1,2]$. According to new estimates from World Health Organization (WHO), there were around 0.48 million new cases of MDR-TB cases, and approximately 0.19 million deaths from MDR-TB worldwide in 2014 (WHO, 2015).

\footnotetext{
*Correspondence: 805722291@qq.com; zhaoyanlin@chinatb.org

${ }^{\dagger}$ Equal contributors

${ }^{2}$ Clinical Laboratory, Chongqing Tuberculosis Control Institute, No. 71, Longteng Street, Jiulongpo District, Chongqing 400050, People's Republic of China

${ }^{3}$ National Center for Tuberculosis Control and Prevention, Chinese Center for Disease Control and Prevention, No. 155, Chang Bai Road, Changping District, Beijing 102206, People's Republic of China

Full list of author information is available at the end of the article
}

Only behind India, China has the second burden of MDRTB globally, with 52, 000 prevalent MDR-TB cases annually (WHO, 2015). A recent national survey of drugresistant tuberculosis in China revealed that $5.7 \%$ of new $\mathrm{TB}$ cases and $25.6 \%$ of previously treated cases were MDR$\mathrm{TB}$, respectively, which were higher than the global average rates [3]. Given its high rate of treatment failure, the epidemic of MDR-TB constitutes a serious public health problem in China [3, 4].

Pyrazinamide (PZA) is one of cornerstone first-line anti-tuberculosis agents that is also commonly used as essential component in the therapeutic treatment of MDR-TB [5, 6]. As the prodrug, PZA requires conversion into its active form pyrazinoic acid (POA) by the enzyme pyrazinamidase (PZase). PZase is encoded by 
the 561-nucleotide pncA gene. Loss of PZase activity cause by genetic mutations in the $p n c A$ gene is the main mechanism of resistance to PZA in M. tuberculosis [6]. Several recent literatures have reported that several PZA-resistant $M$. tuberculosis isolates harbored the mutations in the promoter of $p n c A$ gene, indicating these alternations may result in PZA resistance by influencing the expression of $p n c A$ [7-9]. In addition to $p n c A$, another gene named rpsA, which encodes the $30 \mathrm{~S}$ ribosomal protein S1, has been demonstrated to confer PZA resistance by structural alternation of POA binding [10]. To date, the contribution of the rpsA mutations conferring PZA resistance is controversial, which requires more experimental evidences to elucidate the role of rpsA as a PZA resistance mechanism [8].

Chongqing is the largest municipality in the southwestern China [11]. Due to the underdeveloped setting, Chongqing is considered as a hotspot for both TB and MDR-TB in China [12]. However, limited data is available for the prevalence and molecular characteristics of PZA resistance in $M$. tuberculosis isolates, especially MDR-TB. In this study, our main goal was to investigate the prevalence of PZA resistance among MDR-TB isolates collected in Chongqing municipality. We also sought to analyze the mutant profiles of MDR-TB isolates conferring PZA resistance in this area.

\section{Methods}

\section{Patient enrollment}

All smear-positive tuberculosis patients who were registered at local TB dispensaries between November 2014 and February 2016 were enrolled in this study. Information was obtained from patient's medical record. Two sputum samples were obtained from each smear-positive patient for culturing in the county-level laboratories. After 4-8 weeks of incubation, cultures with growing colonies on Löwenstein-Jensen (L-J) medium were sent to the Chongqing Tuberculosis Control Institute for further drug susceptibility testing. Re-treated cases were defined as patients having previously received more than one month of anti-TB treatment.

\section{Drug susceptibility testing}

Drug susceptibility testing (DST) was performed with the proportional method recommended by WHO $[3,13]$. The concentrations of drugs in L-J medium were as follows: isoniazid (INH), $0.2 \mu \mathrm{g} / \mathrm{ml}$; rifampicin (RIF), $40 \mu \mathrm{g} / \mathrm{ml}$; ethambutol (EMB), $2 \mu \mathrm{g} / \mathrm{ml}$; streptomycin (SM), $4 \mu \mathrm{g} / \mathrm{ml}$; ofloxacin (OFLX), $2 \mu \mathrm{g} / \mathrm{ml}$; kanamycin (KAN), $30 \mu \mathrm{g} / \mathrm{ml}$; amikacin (AMK), $30 \mu \mathrm{g} / \mathrm{ml}$; capromycin (CAP), $40 \mu \mathrm{g} / \mathrm{ml}$; and protionamide (PTO), $40 \mu \mathrm{g} / \mathrm{ml}$ and p-aminosalicylic acid (PAS) $1 \mu \mathrm{g} / \mathrm{ml}$ [14]. A strain was declared resistant to an antimicrobial agent when the growth rate exceeded $1 \%$ compared with the control. In addition, PZA susceptibility testing was determined with a Bactec MGIT 960 system according to the manufacturer's instructions. The critical concentration of PZA in the liquid medium was $100 \mu \mathrm{g} /$ $\mathrm{ml}$ [8]. The MDR-TB strains were defined as those resistant to both isoniazid and rifampicin. In addition, XDR-TB was defined as MDR-TB resistant to any member of the quinolone family and at least one of the remaining second-line anti-TB injectable drugs [15].

\section{DNA extraction and amplification}

Genomic DNA was extracted from freshly cultured bacteria as previously described [16]. Briefly, the harvested bacteria from the surface of L-J medium were suspended in $500 \mu \mathrm{l}$ Tris-EDTA (TE) buffer and heated in a $95{ }^{\circ} \mathrm{C}$ water bath for $30 \mathrm{~min}$. The crude DNA was used as template for amplification. The fragments of $p n c A$ and $r p s A$ were amplified with the following primers: $p n c A-\mathrm{F} 5^{\prime}$ AACAGTTCATCCCGGTTC-3' and $p n c A-\mathrm{R}$ 5'GCGTCATGGACCCTATATC-3'; rpsA-F 5'-CGGAGCAACCCAACAATA-3' and rpsA-R 5'-GTGGACAGCAACGACTTC-3', respectively [17]. The $50 \mu \mathrm{L}$ PCR mixture was prepared as follows: $25 \mu \mathrm{l} 2 \times$ GoldStar MasterMix (CWBio, Beijing, China), $5 \mu \mathrm{L}$ of DNA template, and $0.2 \mu \mathrm{M}$ of each primer set. PCR parameters for amplification were $5 \mathrm{~min}$ at $94{ }^{\circ} \mathrm{C}$ followed by 35 cycles of $94{ }^{\circ} \mathrm{C}$ for $1 \mathrm{~min}, 58{ }^{\circ} \mathrm{C}$ for $1 \mathrm{~min}, 72{ }^{\circ} \mathrm{C}$ for $1 \mathrm{~min}$ and a final extension of $72{ }^{\circ} \mathrm{C}$ for $5 \mathrm{~min}$. PCR products were sent to Tsingke company for sequencing. All sequence data were aligned with pncA and rpsA of reference strain H37Rv (ATCC) using BioEdit (version 7.1.3.0) software.

\section{Quantitative reverse transcription PCR (QRT-PCR)}

Bacteria were harvested from L-J medium after inoculation for 4 weeks. The total RNA was isolated following a standard Trizol RNA extraction protocol supplied by Invitrogen (Invitrogen, Life Technologies, USA) [18]. Followed by treatment with DNaseI (Invitrogen, Life Technologies, USA), the reverse transcription was carried out using SuperScriptIII RT kit (Invitrogen, Life Technologies, USA). The relative expression level of $p n c A$ gene was detected by QRT-PCR in a $20 \mu \mathrm{l}$ system containing $10 \mu \mathrm{L}$ of $2 \times$ UltraSYBR Mixture (CWBio, Beijing, China), $2 \mu \mathrm{L}$ of cDNA template, and $0.2 \mu \mathrm{M}$ of each primer set (pncAQF 5'-GAAGCGGCGGACTACCATC-3' and pncA-QR 5'-AGTGGCGTGCCGTTCTCG-3'). PolyA was set as the internal control in respective PCR experiments [18].

\section{Data analysis}

Chi square test was used to evaluate the associations among multiple categorical variables, and the statistical results were summarized with odds ratios (ORs) with 95\% confidence intervals (CIs). All calculations were performed in SPSS 13.0 (SPSS Inc., USA). Differences with a $P$ value less than 0.05 were declared statistically significant. 


\section{Results}

Demographic characteristics and drug susceptibility profiles A total of 133 (10.8\%) of 1236 clinical isolates were identified as MDR-TB, including 38 (28.6\%) pre-XDR and 17 (12.8\%) XDR. Overall, 80 (60.2\%) strains were isolated from male patients and $53(39.8 \%)$ from female patients. The average age of the patients was 46.6 years (range 19-76 years). In addition, $36.8 \%$ of isolates were from new cases, and $63.2 \%$ from re-treated cases. Out of the 133 MDR isolates tested, $97(72.9 \%)$ were resistant to SM, 40 (30.1\%) to EMB, 52 (39.1\%) to OFLX, 20 (15.0\%) to KAN, $16(12.0 \%)$ to AMK, 11 (8.3\%) to CAP and 15 (11.3\%) to PAS (Table 1).

\section{Factors associated with PZA resistance}

As shown in Table 1, 83 (62.4\%) isolates were determined as PZA-resistant by MGIT, while the other 50 (37.6\%) were susceptible to PZA. In regard to the distribution of MDR-TB cases treatment history, the percentage of re-treated MDR-TB patients in the PZA-resistant group was significantly higher than in the PZAsusceptible group (odd ratio (OR) [95\% confidence interval (CI): $3.26[1.55-6.82], P<0.01)$. In contrast, our data revealed no statistically significant difference between the PZA-resistant and PZA-susceptible group in gender and age $(P>0.05)$.

We further analyzed the resistance profiles of other drugs among PZA-resistant and PZA-susceptible $M$. tuberculosis strains. Statistical analysis revealed that SM(83.1\% vs. $56.0 \%, P<0.01)$, OFLX- $(51.8 \%$ vs. $18.0 \%$, $P<0.01)$, KAN- $(22.9 \%$ vs. $2.0 \%, P<0.01)$, AMK(18.1\% vs. $2.0 \%, P=0.01)$, CAP-resistance $(12.0 \%$ vs. $2.0 \%, P=0.05)$, pre-XDR $(36.1 \%$ vs. $16.0 \%, P<0.02)$ and XDR $(19.3 \%$ vs. $2.0 \%, P<0.01)$ were more frequently observed among PZA-resistant isolates compared with PZA-susceptible isolates, while no difference was identified between EMB-resistant and EMB-susceptible strains (31.3\% vs. $28.0 \%, P=0.69)$ (Table 1 ).

\section{Mutations in the pncA and $r p s A$ gene}

A total of 73 out of $83(88.0 \%)$ MDR strains harbored a mutation located in the pncA gene, including 55 (75.3\%, $55 / 73)$ of single nucleotide substitutions and 18 (24.7\%, $18 / 73$ ) of frameshift mutation. As summarized in Table 2, we observed great mutant diversity in $p n c A$ gene, and there were 48 different mutant types conferring PZA

Table 1 Risk factor associate with PZA resistance among 133 MDR $^{a}$ isolates

\begin{tabular}{|c|c|c|c|c|c|}
\hline \multirow[t]{2}{*}{ Characteristics } & \multirow{2}{*}{$\begin{array}{l}\text { No. }(\%) \text { of isolates } \\
(n=133)\end{array}$} & \multicolumn{2}{|c|}{ No. (\%) of isolates } & \multirow{2}{*}{$\begin{array}{l}\text { OR } \\
(95 \% \text { Cl) }\end{array}$} & \multirow[t]{2}{*}{$P$ value } \\
\hline & & $\begin{array}{l}\mathrm{PZA}^{\mathrm{R}} \\
(n=83)\end{array}$ & $\begin{array}{l}\mathrm{PZA}^{\mathrm{S}} \\
(n=50)\end{array}$ & & \\
\hline \multicolumn{6}{|l|}{ Sex } \\
\hline Male & $80(60.2)$ & $47(56.6)$ & $33(66.0)$ & $0.67(0.33-1.39)$ & 0.29 \\
\hline Female & $53(39.8)$ & $36(43.4)$ & $17(34.0)$ & 1.0 (Ref.) & - \\
\hline \multicolumn{6}{|l|}{ Age group } \\
\hline$<30$ & $31(23.3)$ & $18(21.7)$ & $13(26.0)$ & 1.0 (Ref.) & - \\
\hline $30-59$ & $80(60.2)$ & $55(66.3)$ & $25(50.0)$ & $1.59(0.68-3.74)$ & 0.29 \\
\hline$\geq 60$ & $22(16.5)$ & $10(12.0)$ & $12(24.0)$ & $0.60(0.20-1.81)$ & 0.36 \\
\hline \multicolumn{6}{|c|}{ Treatment history } \\
\hline New case & $49(36.8)$ & $22(26.5)$ & $27(54.0)$ & 1.00 (Ref.) & - \\
\hline Re-treated & $84(63.2)$ & $61(73.5)$ & $23(46.0)$ & $3.26(1.55-6.82)$ & $<0.01$ \\
\hline \multicolumn{6}{|l|}{ Resistance to: } \\
\hline SM & $97(72.9)$ & $69(83.1)$ & $28(56.0)$ & 3.87 (1.74-8.63) & $<0.01$ \\
\hline EMB & $40(30.1)$ & $26(31.3)$ & $14(28.0)$ & $1.17(0.54-2.54)$ & 0.69 \\
\hline OFLX & $52(39.1)$ & $43(51.8)$ & $9(18.0)$ & $4.89(2.11-11.35)$ & $<0.01$ \\
\hline KAN & $20(15.0)$ & $19(22.9)$ & $1(2.0)$ & $14.58(1.88-112.44)$ & $<0.01$ \\
\hline AMK & $16(12.0)$ & $15(18.1)$ & $1(2.0)$ & $10.81(1.38-84.58)$ & 0.01 \\
\hline CAP & $11(8.3)$ & $10(12.0)$ & $1(2.0)$ & $6.71(0.83-54.12)$ & 0.05 \\
\hline PAS & $15(11.3)$ & $13(15.7)$ & $2(4.0)$ & $4.46(0.96-20.65)$ & 0.04 \\
\hline Pre-XDR ${ }^{b}$ & $38(28.6)$ & $30(36.1)$ & $8(16.0)$ & $2.97(1.23-7.16)$ & 0.02 \\
\hline$X R^{c}$ & 17 (12.8) & $16(19.3)$ & $1(2.0)$ & $11.70(1.50-91.22)$ & $<0.01$ \\
\hline
\end{tabular}

${ }^{\mathrm{a} M D R}$ is defined as Mycobacterium tuberculosis strain resistant to at least isoniazid and rifampin

bPre-XDR is defined as MDR strain additionally resistant to either ofloxacin or kanamycin, but not both

${ }^{C} \mathrm{XDR}$ is defined as Mycobacterium tuberculosis strain resistant to isoniazid, rifampin, ofloxacin and kanamycin 
Table 2 Mutations of PZA-resistant MDR-TB isolates within pncA

\begin{tabular}{|c|c|c|c|c|c|}
\hline Locus & Position of nucleotide & Nucleotide substitution & Position of amino acid & Amino acid substitution & No. of isolates \\
\hline \multirow[t]{43}{*}{ pncA } & -11 & TAT $\rightarrow$ TGT & -4 & Tyr $\rightarrow$ Cys & 4 \\
\hline & 2 & ATG $\rightarrow$ ACG & 1 & Met $\rightarrow$ Thr & 1 \\
\hline & 20 & $\mathrm{GTC} \rightarrow \mathrm{GGC}$ & 7 & Val $\rightarrow$ Gly & 2 \\
\hline & 24 & $\mathrm{GAC} \rightarrow \mathrm{GAG}$ & 8 & $\mathrm{Asp} \rightarrow \mathrm{Glu}$ & 1 \\
\hline & 28 & $\mathrm{CAG} \rightarrow \mathrm{TAG}$ & 10 & Gln $\rightarrow$ Stop & 1 \\
\hline & 35 & $\mathrm{GAC} \rightarrow \mathrm{GCC}$ & 12 & $\mathrm{Asp} \rightarrow \mathrm{Ala}$ & 2 \\
\hline & 37 & $\mathrm{TTC} \rightarrow \mathrm{GTC}$ & 13 & Phe $\rightarrow$ Val & 1 \\
\hline & 40 & $\mathrm{TGC} \rightarrow \mathrm{CGC}$ & 14 & Cys $\rightarrow$ Arg & 2 \\
\hline & 40 & $\mathrm{TGC} \rightarrow \mathrm{CGC}$ & 14 & Cys $\rightarrow$ Arg & 1 \\
\hline & 94 & $\mathrm{TTC} \rightarrow \mathrm{GTC}$ & 32 & Phe $\rightarrow$ Val & 1 \\
\hline & 123 & $\mathrm{TAC} \rightarrow \mathrm{TAG}$ & 41 & Tyr $\rightarrow$ Stop & 1 \\
\hline & 146 & $\mathrm{GAC} \rightarrow \mathrm{GCC}$ & 49 & Asp $\rightarrow$ Gly & 1 \\
\hline & 146 & $\mathrm{GAC} \rightarrow \mathrm{GCC}$ & 49 & $\mathrm{Asp} \rightarrow \mathrm{Ala}$ & 2 \\
\hline & 151 & $\mathrm{CAC} \rightarrow \mathrm{TAC}$ & 51 & $\mathrm{His} \rightarrow \mathrm{Tyr}$ & 1 \\
\hline & 151 & $\mathrm{CAC} \rightarrow \mathrm{CGC}$ & 51 & $\mathrm{His} \rightarrow \mathrm{Arg}$ & 1 \\
\hline & 152 & $\mathrm{CAC} \rightarrow \mathrm{CCC}$ & 51 & $\mathrm{His} \rightarrow$ Pro & 1 \\
\hline & 170 & $\mathrm{CAC} \rightarrow \mathrm{CGC}$ & 57 & $\mathrm{His} \rightarrow \mathrm{Arg}$ & 1 \\
\hline & 185 & $\mathrm{CCG} \rightarrow \mathrm{CTG}$ & 62 & Pro $\rightarrow$ Leu & 5 \\
\hline & 206 & $\mathrm{CCG} \rightarrow \mathrm{CTG}$ & 69 & Pro $\rightarrow$ Leu & 1 \\
\hline & 213 & $\mathrm{CAT} \rightarrow \mathrm{CAG}$ & 71 & $\mathrm{His} \rightarrow \mathrm{Gln}$ & 1 \\
\hline & 226 & $\mathrm{ACT} \rightarrow \mathrm{CCT}$ & 76 & Thr $\rightarrow$ Pro & 3 \\
\hline & 232 & $\mathrm{GGC} \rightarrow \mathrm{AGC}$ & 78 & Gly $\rightarrow$ Asp & 1 \\
\hline & 245 & $\mathrm{CAT} \rightarrow \mathrm{CGT}$ & 82 & $\mathrm{His} \rightarrow \mathrm{Arg}$ & 1 \\
\hline & 286 & $\mathrm{AAG} \rightarrow \mathrm{CAG}$ & 96 & Lys $\rightarrow$ Gln & 1 \\
\hline & 307 & $\mathrm{TAC} \rightarrow \mathrm{CAC}$ & 103 & $\mathrm{Tyr} \rightarrow \mathrm{His}$ & 1 \\
\hline & 309 & $\mathrm{TAC} \rightarrow \mathrm{TAG}$ & 103 & Tyr $\rightarrow$ Stop & 1 \\
\hline & 319 & $\mathrm{GAA} \rightarrow \mathrm{AAA}$ & 107 & Glu $\rightarrow$ Lys & 1 \\
\hline & 395 & $\mathrm{GGT} \rightarrow \mathrm{GAT}$ & 132 & Gly $\rightarrow$ Asp & 9 \\
\hline & 425 & $\mathrm{ACG} \rightarrow \mathrm{ATG}$ & 142 & Thr $\rightarrow$ Met & 1 \\
\hline & 437 & $\mathrm{GCG} \rightarrow \mathrm{GTG}$ & 146 & $\mathrm{Ala} \rightarrow \mathrm{Val}$ & 1 \\
\hline & 464 & $\mathrm{GTG} \rightarrow \mathrm{GGG}$ & 155 & Val $\rightarrow$ Gly & 1 \\
\hline & 488 & $\mathrm{GTG} \rightarrow \mathrm{GCG}$ & 163 & $\mathrm{Val} \rightarrow \mathrm{Ala}$ & 1 \\
\hline & 515 & $\mathrm{CTG} \rightarrow \mathrm{CCG}$ & 172 & Leu $\rightarrow$ Pro & 2 \\
\hline & 52 & insertion of GC & & & 1 \\
\hline & 130 & insertion of $C$ & & & 1 \\
\hline & 136 & deletion of G & & & 2 \\
\hline & 139 & insertion of CA & & & 1 \\
\hline & 232 & insertion of $C$ & & & 1 \\
\hline & 243 & insertion of $\mathrm{T}$ & & & 1 \\
\hline & 288 & insertion of $A$ & & & 1 \\
\hline & 341 & deletion of ACGCC & & & 1 \\
\hline & 342 & deletion of GCCAC & & & 2 \\
\hline & 376 & deletion of GATGAGGTC & & & 1 \\
\hline & 392 & insertion of G & & & 1 \\
\hline
\end{tabular}


Table 2 Mutations of PZA-resistant MDR-TB isolates within pncA (Continued)

\begin{tabular}{|c|c|c|c|c|c|}
\hline Locus & Position of nucleotide & Nucleotide substitution & Position of amino acid & Amino acid substitution & No. of isolates \\
\hline & 392 & insertion of GG & & & 1 \\
\hline & 393 & insertion of GGT & & & 1 \\
\hline & 408 & insertion of CA & & & 1 \\
\hline & 408 & insertion of $\mathrm{A}$ & & & 2 \\
\hline & \multicolumn{4}{|c|}{ Total } & 73 \\
\hline
\end{tabular}

resistance among MDR strains in Chongqing. The most prevalent mutation associated with PZA resistance was found in codon 132 of pncA (12.3\%, 9/73), resulting in the amino aicd substitution of Gly to Asp. As the second affected codon, five isolates had a mutation in codon 62 $(6.8 \%, 5 / 73)$. Notably, the third frequent mutation was the nucleotide substitution from $\mathrm{A}$ to $\mathrm{G}$ at position -11 in the promoter region of $p n c A$, accounting for $5.4 \%$ of mutant isolates. We also found that 4 PZA-susceptible isolates carried a genetic mutation in $p n c A$, including 2 strains in codon 62 , one in codon 67 and one in codon 154 (Table 3). In addition, no genetic mutation associated with PZA resistance was found in the rpsA gene in this study.

We analyzed the performance of $p n c A$ mutations for predicting PZA susceptibility. When setting the phenotypic PZA susceptibility as a gold standard, we found that detection of mutations in $p n c A$ gene exhibited a sensitivity of $88.0 \%(95 \% \mathrm{CI}, 80.9 \%-95.0 \%)$ and a specificity of $92.0 \%(95 \%$ CI, $84.5 \%-99.5 \%)$ (Table 4$)$.

\section{Loss of pncA expression due to promoter mutation}

We further explore whether the substitution at position -11 affected the expression level of $p n c A$. As shown in Fig. 1, compared with reference strain $H 37 R v$, the relative expression level of pncA in MDR87, MDR88, MDR114 and MDR126 carried the substitution at position -11 were 0.25 fold, 0.19 fold, 0.36 fold and 0.22 fold, respectively. Statistical analysis revealed that the $p n c A$ expression of strains harboring promoter mutation at position -11 was significantly lower than that of $\operatorname{H} 37 \operatorname{Rv}(P<0.01)$.

\section{Discussion}

PZA plays a unique role in the treatment for MDR-TB in both first- and second-line regimens $[19,20]$. The prevalence of PZA among MDR-TB thus is a determining factor for initiation of PZA in the therapy regimens for these refractory patients [21]. Here, our data demonstrated that $62.4 \%$ of MDR-TB exhibited resistance against PZA in Chongqing, which was similar to a recent literature from Beijing (57.7\%) [22], while higher than those from Zhejiang (43.1\%) [8], Shanghai (38.5\%) [23], United States (38.0\%) [19], and Thailand (49.0\%) [24]. The high prevalence of PZA resistance among MDR-TB patients from our report indicates that Chongqing is a hotspot of PZA resistance in China. In our study, more than $60 \%$ MDR patients received previous anti-TB therapy with PZA, which is significantly higher than the average national level (21.8\%) [3]. Hence, we speculate that the high proportion of PZA resistance may be contributed to the high rate of re-treated TB patients. The serious issue on PZA resistance highlights the diminished role of PZA in the treatment for MDR-TB in this setting with high MDR-TB burden. Prior to the use of PZA for treatment of MDR-TB cases, it is essential to perform in vitro susceptibility testing against PZA to formulate a suitable regimen [21].

Another important finding from our observation was that we observed that there were high correlation between PZA resistance and several other drugs' resistance, including OFLX, second-line injectable drugs, and PAS. Similar to our findings, a recent report from Alame-Emane and colleagues has revealed that PZA resistance in $M$. tuberculosis arises after RIF and fluoroquinolone (FQ) resistance [25]. Genetic mutations constitute the most important mechanism conferring drug resistance in M. tuberculosis [20]. Exposure to bacterial species to antimicrobial agents, including RIF, FQ and the aminoglycosides, induces the production of oxygen radicals, thereby conferring high frequency mutagenesis [25-27]. Considering long duration of anti-TB

Table 3 Mutations in PZA-susceptible MDR-TB isolates within pncA gene

\begin{tabular}{lllllc}
\hline Locus & Position of nucleotide & Nucleotide substitution & Position of amino acid & Amino acid substitution & No. of isolates \\
\hline pncA & 184 & CCG $\rightarrow$ ACG & 62 & Pro $\rightarrow$ Thr & 1 \\
& 185 & CCG $\rightarrow$ CAG & 62 & Pro $\rightarrow$ Gln & 1 \\
& 200 & TCG $\rightarrow$ TGG & 67 & Ser $\rightarrow$ Trp & 1 \\
& AG $\rightarrow$ AAG & Arg $\rightarrow$ Lys & 1 & 4 \\
\hline
\end{tabular}


Table 4 Performance of pncA mutations for predicting PZA susceptibility

\begin{tabular}{|c|c|c|c|c|c|c|c|}
\hline \multirow{2}{*}{$\begin{array}{l}\text { Mutation } \\
\text { in pncA }\end{array}$} & \multicolumn{2}{|c|}{ PZA susceptibility ${ }^{a}$} & \multirow[t]{2}{*}{ Total } & \multirow{2}{*}{$\begin{array}{l}\text { Sensitivity } \\
(95 \% \mathrm{Cl}, \%)\end{array}$} & \multirow{2}{*}{$\begin{array}{l}\text { Specificity } \\
(95 \% \mathrm{Cl}, \%)\end{array}$} & \multirow{2}{*}{$\begin{array}{l}\text { PPV } \\
(95 \% \mathrm{Cl}, \%)\end{array}$} & \multirow{2}{*}{$\begin{array}{l}\text { NPV } \\
(95 \% \mathrm{Cl}, \%)\end{array}$} \\
\hline & $\mathrm{R}$ & S & & & & & \\
\hline Yes & 73 & 4 & 77 & \multirow{3}{*}{$\begin{array}{c}88.0 \\
(80.9-95.0)\end{array}$} & \multirow{3}{*}{$\begin{array}{c}92.0 \\
(84.5-99.5)\end{array}$} & \multirow{3}{*}{$\begin{array}{c}94.8 \\
(89.8-99.8)\end{array}$} & \multirow{3}{*}{$\begin{array}{c}82.1 \\
(72.1-92.2)\end{array}$} \\
\hline No & 10 & 46 & 56 & & & & \\
\hline Total & 83 & 50 & 133 & & & & \\
\hline
\end{tabular}

${ }^{a} R$, resistant, $S$ susceptible, $P P V$ positive predictive value, $N P V$ negative predictive value, $C l$ confidence interval

treatment, we hypothesize that MDR bacteria will harbor more genetic mutations induced by prolonged exposure to these drugs, which may be responsible for the potential cross resistance between PZA and other drugs in our study.

In vitro susceptibility against PZA is essential for proper management of MDR-TB with regimen containing PZA [21]. However, phenotypic DST for PZA is not routinely performed due to the requirement of harshly acidic environment [17]. Molecular method based on detecting the mutations in $p n c A$ and $r p s A$ serves as an alternative to predict the PZA susceptibility in $M$. tuberculosis [9]. In this study, our data demonstrated that genetic alternations in $p n c A$ confer $88.0 \%$ of PZA resistance among MDR-TB in Chongqing. A number of studies have demonstrated a diverse prevalence of $p n c A$ mutation among PZA resistant isolates in different regions, ranging from $45.7 \%$ in Brazil [28], $70.6 \%$ in Iran [29], 75.0\% in Thailand [24], 78.0\% in Zhejiang [8], $84.6 \%$ in Southern China [9], and 94.1\% in Sweden [30]. Hence, $p n c A$ mutations may differ from one geographic region to another. In addition, we found that $p n c A \mathrm{mu}-$ tations exhibited great diversity, and the most frequent mutant type in codon 142 only accounted for approximate $12 \%$ of PZA resistant isolates, which was also different from reports from other regions $[8,9]$. Given the diversity of $p n c A$ mutations within more than 500-bp long segment, DNA sequencing of the entire $p n c A$ is more effective for verification of PZA resistance rather than the routine methods by covering the mutant hotspots.

In addition, the third frequent mutation identified in this study was located at position -11 of the $p n c A$ promoter region. In line with our observation, numerous literatures have observed this mutant type in PZAresistant $M$. tuberculosis isolates [31, 32]. We found that this substitution at position -11 was associated with low level of $p n c A$ expression, which was also consistent to the observation from Sheen et al. [31]. The anti-TB activity of PZA depends on the transformation to POA by PZAse, which is encoded by $p n c A$ gene. The loss of $p n c A$ transcriptional level may result in the relative low PZAse activity, which is further associated with the phenotypic PZA resistance. Our results suggest that the promoter region of $p n c A$ is recommended to be included in the sequence analysis of $p n c A$ gene.

We acknowledge several limitations of this study. First, the small sample size is a major limitation of our report. And all the strains collected from one region also reduce their representativeness and thereby constrict the generalizability of findings. Further wider sampling will give more credence to this study. Second, although the primary data of this study suggest that loss of $p n c A$ expression caused by promoter mutation confers PZA resistance in MDR-TB isolates, we could give no biochemical or transgenic substantiation of this statement. Therefore, there is an urgent need to confirm our findings with more experimental evidences in the future.

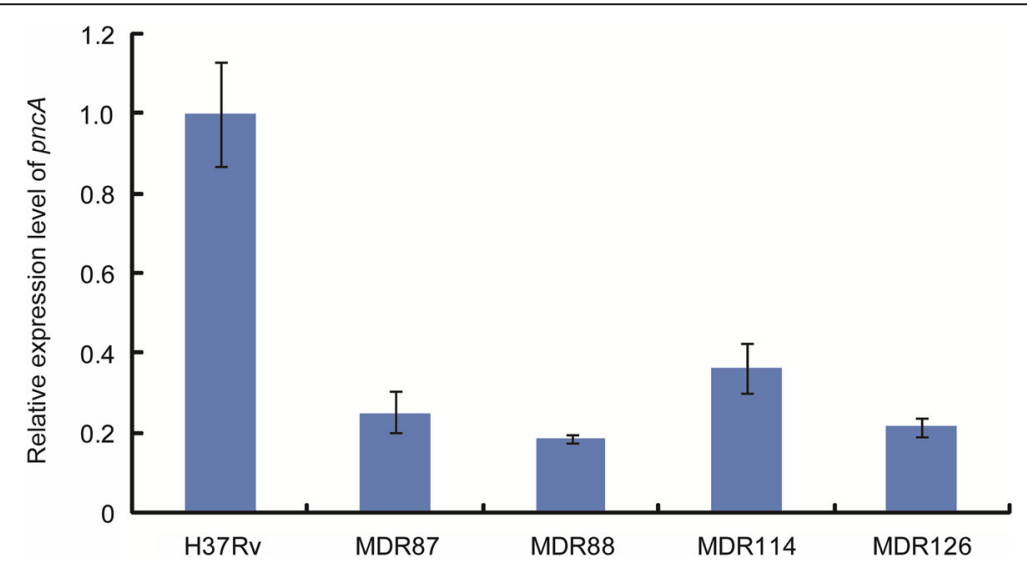

Fig. 1 Relative expression level of pncA gene in 4 M. tuberculosis isolates with mutation in the position -11 of promoter region 
Third, the high diversity of $p n c A$ mutations in MTB-TB isolates from Chongqing underscores previous findings that there is no clear hotspot for pncA mutations [30], and several novel mutations in $p n c A$ gene were found for first time among PZA-resistant isolates. Despite being highly correlated with the loss of PZA susceptibility, further experiments will be carried out to clarify the potential contributions of these mutations to PZA resistance. Nevertheless, our report firstly described the molecular characteristics of PZA resistance among MDR-TB isolates from Southern China, which provides important hints to diagnose PZA resistance and help guide therapy with PZA for MDR-TB patients in this region with high MDR-TB burden.

\section{Conclusion}

In conclusion, our data have demonstrated that the analysis of the pncA gene rather than rpsA gene provides rapid and accurate information regarding PZA susceptibility for MDR-TB isolates in Chongqing. In addition, loss of pncA expression caused by promoter mutation confers PZA resistance in MDR-TB isolates. Considering the high degree of diversity of $p n c A$ gene mutations, DNA sequencing of the entire $p n c A$ is more effective for verification of PZA resistance rather than the routine methods by covering the mutant hotspots. The high prevalence of PZA resistance among MDR highlights the diminished role of PZA in the treatment for MDRTB in this setting with high TB burden.

\section{Abbreviations \\ AMK: Amikacin; CAP: Capromycin; Cls: Confidence intervals; DST: Drug susceptibility testing; EMB: Ethambutol; INH: Isoniazid; KAN: Kanamycin; MDR- TB: Multidrug-resistant tuberculosis; OFLX: Ofloxacin; ORs: Odds ratios; PAS: P- aminosalicylic acid; POA: Pyrazinoci acid; PTO: Protionamide; \\ PZA: Pyrazinamide; PZase: Pyrazinamidase; RIF: Rifampicin; SM: Streptomycin}

\section{Acknowledgements}

We would like to thank the staffs from the National Tuberculosis Reference Laboratory for their technical support.

\section{Funding}

This study was supported by the Major State Basic Research Development Program of China (2014CB744403)

\section{Availability of data and materials}

The datasets used and/or analysed during the current study available from the corresponding author on reasonable request.

\section{Authors' contributions}

Conceived and designed the experiments: YP, DZ, JL, YL. Performed the experiments: YP, DZ, HZ, JS. YH, JL. Analyzed the data: YP, DZ, JL, YL. Wrote the paper: YP, DZ, JL, YL. All authors contributed to the final manuscript. All authors read and approved the final manuscript.

\section{Ethics approval and consent to participate}

This study was approved by the Ethics Committee of the Chongqing Tuberculosis Control Institute. Patients enrolled in this study provided the signed Informed Consent form.

\section{Consent for publication}

Not applicable.

\section{Competing interests}

The authors declare that they have no competing interests.

\section{Publisher's Note}

Springer Nature remains neutral with regard to jurisdictional claims in published maps and institutional affiliations.

\section{Author details}

${ }^{1}$ National Clinical Laboratory on Tuberculosis, Beijing Key Laboratory on Drug-Resistant Tuberculosis Research, Beijing Chest Hospital, Capital Medical University, Beijing Tuberculosis and Thoracic Tumor Institute, Beijing, China. ${ }^{2}$ Clinical Laboratory, Chongqing Tuberculosis Control Institute, No. 71, Longteng Street, Jiulongpo District, Chongqing 400050, People's Republic of China. ${ }^{3}$ National Center for Tuberculosis Control and Prevention, Chinese Center for Disease Control and Prevention, No. 155, Chang Bai Road, Changping District, Beijing 102206, People's Republic of China.

Received: 26 February 2017 Accepted: 22 September 2017

Published online: 06 November 2017

\section{References}

1. Gandhi NR, Nunn P, Dheda K, Schaaf HS, Zignol M, van Soolingen D, Jensen $P$, Bayona J. Multidrug-resistant and extensively drug-resistant tuberculosis: a threat to global control of tuberculosis. Lancet. 2010;375(9728):1830-43.

2. Zhang Z, Pang Y, Wang Y, Liu C, Zhao Y. Beijing genotype of Mycobacterium tuberculosis is significantly associated with linezolid resistance in multidrug-resistant and extensively drug-resistant tuberculosis in China. Int J Antimicrob Agents. 2014;43(3):231-5.

3. Zhao Y, Xu S, Wang L, Chin DP, Wang S, Jiang G, Xia H, Zhou Y, Li Q, Ou X, et al. National survey of drug-resistant tuberculosis in China. N Engl J Med. 2012;366(23):2161-70

4. Orenstein EW, Basu S, Shah NS, Andrews JR, Friedland GH, Moll AP, Gandhi NR, Galvani AP. Treatment outcomes among patients with multidrugresistant tuberculosis: systematic review and meta-analysis. Lancet Infect Dis. 2009:9(3):153-61.

5. Verdugo D, Fallows D, Ahuja S, Schluger N, Kreiswirth B, Mathema B. Epidemiologic Correlates of Pyrazinamide-Resistant Mycobacterium tuberculosis in New York City. Antimicrob Agents Chemother. 2015;59(10): 6140-50.

6. Zhang $Y$, Mitchison D. The curious characteristics of pyrazinamide: a review. Int J Tuberc Lung Dis. 2003;7(1):6-21.

7. Scorpio A, Zhang Y. Mutations in pncA, a gene encoding pyrazinamidase/ nicotinamidase, cause resistance to the antituberculous drug pyrazinamide in tubercle bacillus. Nat Med. 1996;2(6):662-7.

8. Xia Q, Zhao LL, Li F, Fan YM, Chen YY, Wu BB, Liu ZW, Pan AZ, Zhu M. Phenotypic and genotypic characterization of pyrazinamide resistance among multidrug-resistant Mycobacterium tuberculosis isolates in Zhejiang. China Antimicrob Agents Chemother. 2015;59(3):1690-5.

9. Tan Y, Hu Z, Zhang T, Cai X, Kuang H, Liu Y, Chen J, Yang F, Zhang K, Tan S, et al. Role of pncA and rpsA gene sequencing in detection of pyrazinamide resistance in Mycobacterium tuberculosis isolates from southern China. J Clin Microbiol. 2014;52(1):291-7.

10. Shi W, Zhang X, Jiang X, Yuan H, Lee JS, Barry CE 3rd, Wang H, Zhang W, Zhang Y. Pyrazinamide inhibits trans-translation in Mycobacterium tuberculosis. Science. 2011;333(6049):1630-2.

11. Zhang D, An J, Wang J, Hu C, Wang Z, Zhang R, Wang Y, Pang Y. Molecular typing and drug susceptibility of Mycobacterium tuberculosis isolates from Chongqing Municipality, China. Infect Genet Evol. 2013;13:310-6.

12. Zhang D, An J, Wang Y, Pang Y. Genetic diversity of multidrug-resistant tuberculosis in a resource-limited region of China. Int J Infect Dis. 2014:29:7-11.

13. World Health Organization: Global tuberculosis report 2015. Geneva: World Health Organization; 2015

14. World Health Organization. Anti-tuberculosis drug resistance in the world Geneva: World Health Organization; 2008.

15. Pang Y, Zhou Y, Zhao B, Liu G, Jiang G, Xia H, Song Y, Shang Y, Wang S, Zhao YL. Spoligotyping and drug resistance analysis of Mycobacterium tuberculosis strains from national survey in China. PLoS One. 2012;7(3): e32976. 
16. Zhang Z, Lu J, Wang Y, Pang Y, Zhao Y. Prevalence and molecular characterization of fluoroquinolone-resistant Mycobacterium tuberculosis isolates in China. Antimicrob Agents Chemother. 2014;58(1):364-9.

17. Pang $Y$, Wang $Z$, Zheng $H$, Song $Y$, Wang $Y$, Zhao $Y$. Pyrazinamide resistance determined by liquid culture at low pH better correlates with genetic mutations in MDR tuberculosis isolates. J Microbiol Methods. 2015;119:142-4.

18. Pang Y, Lu J, Wang Y, Song Y, Wang S, Zhao Y. Study of the rifampin monoresistance mechanism in Mycobacterium tuberculosis. Antimicrob Agents Chemother. 2013;57(2):893-900.

19. Kurbatova EV, Cavanaugh JS, Dalton T, Click ES, Cegielski JP. Epidemiology of pyrazinamide-resistant tuberculosis in the United States, 1999-2009. Clin Infect Dis. 2013;57(8):1081-93.

20. Zhang Y, Yew WW. Mechanisms of drug resistance in Mycobacterium tuberculosis: update 2015. Int J Tuberc Lung Dis. 2015;19(11):1276-89.

21. Zhang Y, Chiu Chang K, Leung CC, Wai Yew W, Gicquel B, Fallows D, Kaplan G, Chaisson RE, Zhang W. 'Z(S)-MDR-TB' versus 'Z(R)-MDR-TB': improving treatment of MDR-TB by identifying pyrazinamide susceptibility. Emerg Microbes Infect. 2012;1(7):e5.

22. Gu Y, Yu X, Jiang G, Wang X, Ma Y, Li Y, Huang H. Pyrazinamide resistance among multidrug-resistant tuberculosis clinical isolates in a national referral center of China and its correlations with pncA, rpsA, and panD gene mutations. Diagn Microbiol Infect Dis. 2016;84(3):207-11.

23. Xu P, Wu J, Yang C, Luo T, Shen X, Zhang Y, Nsofor CA, Zhu G, Gicquel B, Gao $Q$. Prevalence and transmission of pyrazinamide resistant Mycobacterium tuberculosis in China. Tuberculosis (Edinb). 2016;98:56-61.

24. Jonmalung J, Prammananan T, Leechawengwongs M, Chaiprasert A. Surveillance of pyrazinamide susceptibility among multidrug-resistant Mycobacterium tuberculosis isolates from Siriraj Hospital. Thailand BMC Microbiol. 2010;10:223.

25. Alame-Emane AK, Xu P, Pierre-Audigier C, Cadet-Daniel V, Shen X, Sraouia M, Siawaya JF, Takiff H, Gao Q, Gicquel B. Pyrazinamide resistance in Mycobacterium tuberculosis arises after rifampicin and fluoroquinolone resistance. Int J Tuberc Lung Dis. 2015;19(6):679-84.

26. Ysern P, Clerch B, Castano M, Gibert I, Barbe J, Llagostera M. Induction of SOS genes in Escherichia coli and mutagenesis in Salmonella typhimurium by fluoroquinolones. Mutagenesis. 1990;5(1):63-6.

27. Baharoglu Z, Mazel D. Vibrio cholerae triggers SOS and mutagenesis in response to a wide range of antibiotics: a route towards multiresistance. Antimicrob Agents Chemother. 2011:55(5):2438-41.

28. Bhuju S, Fonseca Lde S, Marsico AG, de Oliveira Vieira GB, Sobral LF, Stehr M, Singh M, Saad MH. Mycobacterium tuberculosis isolates from Rio de Janeiro reveal unusually low correlation between pyrazinamide resistance and mutations in the pncA gene. Infect Genet Evol. 2013;19:1-6.

29. Doustdar F, Khosravi AD, Farnia P. Mycobacterium tuberculosis genotypic diversity in pyrazinamide-resistant isolates of Iran. Microb Drug Resist. 2009; 15(4):251-6.

30. Jureen P, Werngren J, Toro JC, Hoffner S. Pyrazinamide resistance and pncA gene mutations in Mycobacterium tuberculosis. Antimicrob Agents Chemother. 2008;52(5):1852-4.

31. Sheen P, Lozano K, Gilman RH, Valencia HJ, Loli S, Fuentes P, Grandjean L, Zimic M. pncA gene expression and prediction factors on pyrazinamide resistance in Mycobacterium tuberculosis. Tuberculosis (Edinb). 2013;93(5): 515-22

32. Portugal I, Barreiro L, Moniz-Pereira J, Brum L. pncA mutations in pyrazinamide-resistant Mycobacterium tuberculosis isolates in Portugal. Antimicrob Agents Chemother. 2004;48(7):2736-8.

\section{Submit your next manuscript to BioMed Central and we will help you at every step:}

- We accept pre-submission inquiries

- Our selector tool helps you to find the most relevant journal

- We provide round the clock customer support

- Convenient online submission

- Thorough peer review

- Inclusion in PubMed and all major indexing services

- Maximum visibility for your research

Submit your manuscript at www.biomedcentral.com/submit
Biomed Central 